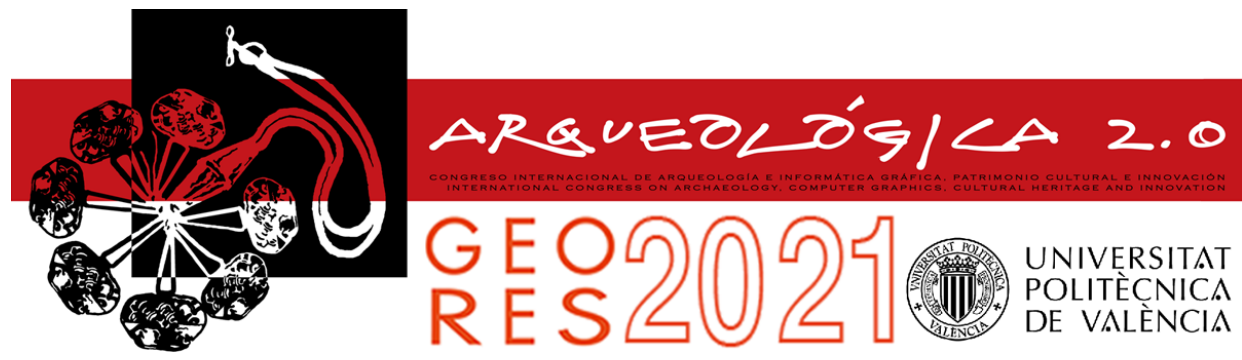

Proceedings of the joint international event $9^{\text {th }}$ ARQUEOLÓGICA

$2.0 \& 3^{\text {rd }}$ GEORES

Valencia (Spain).

26-28 April 2021

\title{
TECHNIQUES OF GEOMATICS AND SOFT COMPUTING FOR THE MONITORING OF UNSAFE BUILDINGS
}

\author{
Ernesto Bernardo a, ${ }^{,}$, Giuliana Bilottab \\ ${ }^{a}$ Department of Civil Engineering, Energy, Environment and Materials (DICEAM), Mediterranea University of Reggio Calabria, Via \\ Graziella Feo di Vito, 89124 Reggio Calabria, Italy. ernesto.bernardo@unirc.it \\ ${ }^{\text {b }}$ Department of Planning, University IUAV of Venice, Santa Croce 191 Tolentini, 30135 Venice, Italy. giuliana.bilotta@iuav.it
}

\begin{abstract}
:
The proposed research activity is part of the conservation of cultural heritage and is based on the study and development of advanced techniques for monitoring, inspecting and mapping building cracks in order to obtain and constantly update the safety status of building through a GIS platform. Data collection (initial and periodic) is one of the most important phases of the monitoring process and allows to obtain information about the integrity of the buildings, essential to plan future design and intervention choices. This can be carried out both through traditional tools (3D laser scanners, GNSS receivers, motorized total station) and innovative tools (such as remote sensing or UAVs). The goal of the research was the design and construction of an innovative automated system for monitoring and continuous data acquisition (big data). Furthermore, we implemented algorithms dedicated to the management of the amount of georeferenced data (big data) acquired. We optimized their representation on GIS (Geographic Information System) platforms in order to obtain an "open and updatable" thematic cartography, and set up a sort of Cadastre of unsafe buildings in the village of Casalvecchio Siculo (possibly extended to other villages in the future). This is intended (in our application) as an updatable IT tool for archiving, viewing, querying and managing all the data that the municipality and the regions have on their own villages. In it, it will be possible to represent the elements inherent to the geometric characteristics of the buildings, their relevance, the state of the cracks, the interventions carried out in the most important historical buildings and the systems created, having databases available that allow quick selective searches by topics.
\end{abstract}

Keywords: UAV, big data, virtual archaeology, digital archaeology, cultural heritage, 3D reconstruction

\section{Introduction}

This note refers to a series of activities developed by the Geomatics Laboratory of the Mediterranean University of Reggio Calabria in the context of Cultural Heritage (Bernardo, Musolino, \& Maesano, 2021) with particular reference to the monitoring, diffusion, dissemination of small villages. In particular, attention is focused on the development of a complex innovative and automated system capable of acquiring, processing and managing images by treating them with soft computing techniques in order to extract significant details of the area object of the image, transposing the results automatically into a GIS. These activities are experiments currently in progress within a PhD thesis that is part of the update of the Road Cadastre entitled: "Geomatics and soft computing techniques for monitoring infrastructure and managing big data".

For several decades, the introduction of electronic computers has led to a substantial revolution in the ability to analyze and collect data, a field that until now perhaps has not fully benefited from the latest technological advances of the digital age (Postorino, Barrile, \& Cotroneo, 2006). In practice, what used to be acquired in the field manually with punctual campaigns and consequently with relatively high time and cost, perhaps while leveraging advanced technologies, can now be made available through a growing number of devices such as sensors, actuators and cameras, characterized by ever-increasing automation (also in terms of the possibility of a subsequent integrated and dynamic analysis of the data) and increasingly reduced costs (Barrile, Bernardo, \& Fotia, 2020).

Among the many types of sensors that can be used in the monitoring of the building are already today consolidated examples of hardware systems aimed at quantifying virtually real-time, or almost, state of the crack, geometric elements of a building (for example, point clouds by laser scanning), etc (Shan \& Toth, 2018; Chen, Truong-Hong, Laefer, \& Mangina, 2018; Florkova, Duris, Veselovsky, Sedivý, \& Kovalova, 2018).

It will be possible to carry out visual inspections of the state of a building in a continuous and automatic way by drones, ground and air, using for the analysis of the 
collected data (in the form of instrumental measurements, but also images) of artificial intelligence, overcoming in this the intrinsic limits of a human operator in terms of fallibility and ability to compare a large amount of data variable over time.

The first effect of this evolution is the increase in the amount of building information that can be stored in databases, whose availability and number for stakeholders is growing at an exponential rate, also introducing the new aspect of dynamic data over time. At the same time, even traditional analytics and management systems are beginning to be insufficient to process such a large amount of big data (Bernardo, Barrile, \& Fotia, 2020). The archives of the managing bodies also contain the register of works, the history of maintenance interventions with the relative costs and all information related to the safety of important buildings. Therefore, one of the main elements of building network management becomes the punctual and predictive query and analysis system used to manage the database in order to arrive at informed decisions on the interventions to be carried out, also implementing decisions in the most real-time possible.

Although the amount of data can be considered a distinctive character, the heterogeneity and structure of the information strongly characterize this type of data and, even more so, it becomes essential to be able to rely on effective methods and as automated as possible, which enhance only the information useful for analysis, discarding unnecessary information to avoid overburdening the system, but without the risk of incurring in oversimplification or amplification of errors.

The biggest challenge of big data management lies in the development of methods capable of predicting future observations, optimize the information available, and at the same time find correlations between the information collected (Bernardo, Barrile, Fotia, \& Bilotta 2020). In this regard (management and use of the large amount of data acquired for building monitoring), the application reported in this note can be particularly useful. In fact, the aim of the research will be the design and implementation of an innovative system of measurement sensors and components to be equipped on technological data acquisition systems.

The research also consists of the implementation of algorithms dedicated to the management of the amount of georeferenced data obtained and their representation on GIS (Geographic Information System) platforms as "open and updatable" thematic cartography. These activities are in experimentation for a PhD thesis. This developed system is therefore an open GIS to be considered as a valid tool also for updating the Cadastre of unsafe Buildings; once completed and implemented it can be proposed to the Authorities responsible for its use.

\section{Materials and methods}

\subsection{Study Area}

We decided to test the experimental methodology on the town of Castelvecchio Siculo, an Italian municipality in the metropolitan city of Messina, one of the largest in eastern Sicily. The town, located at $420 \mathrm{~m}$. above sea level, on the slopes of Mount Sant'Elia, dominates the Valle d'Agrò and represents a reference point for cultural, artistic, architectural and naturalistic tourism. According to an Aragonese deed of 1351, the original Greek-Byzantine name of the town was "Palachorion" (Old Casale), a name which, over time, was translated into the Latin language "Rus Vetus", "Casale Vetus" and the current Casalvecchio. From the beginning of the Norman age until the eighteenth century it was under the jurisdiction of Savoca, from 1928 until 1939 a hamlet of Santa Teresa di Riva, finally an autonomous municipality (Fig. 1). There are numerous historical buildings of high quality.

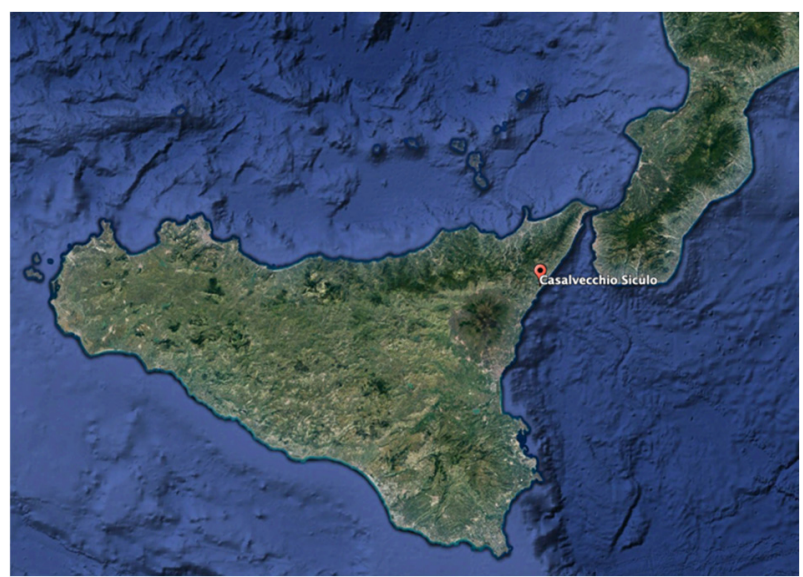

Figure 1: Casalvecchio Siculo, (ME), Sicily, Italy.

\subsection{Cadastre of unsafe buildings and proposed automatic update methodology}

The system we propose provides a fleet of drones that in fully automatic mode takes off and acquires images of the buildings to be monitored, then the images of the buildings are processed in order to highlight any damage present, also obtaining a 3D model of the same. The 3D models are transferred into an updatable GIS to obtain the safety level of the buildings according to the detected cracks. Keeping in mind the possible evolution of the cracks over time, the proposed system will allow the updating of 3D models by also implementing additional useful information characteristic of the building. These 3D models will be updated from time to time by implementing other useful information (architectural details of particular value, interior plans, links to view or download the BIM model). In particular, in this paper, we will focus on the experimental methodology used for the identification of cracks and therefore for the level of safety in the GIS by updating the attributes of the vector file with the results obtained from the elaboration of the methodology produced (Barrile \& Cotroneo, 2006).

The Cadastre of unsafe buildings of the village could have as its first objective to represent all the elements inherent to the geometric characteristics of the buildings, their relevance, the state of the cracks, the interventions carried out in the most important historic buildings and the systems created having databases available that allow fast selective searches by subjects.

The segmented attributes of elements could be grouped into homogeneous entities. Among these, the most significant could be: (1) cracks; (2) presence of exposed iron; (3) presence of humidity; (4) plaster damage; (5) construction type.

In this case, we experimented with an innovative procedure that involves the use of a properly designed 
fleet of drones that, proceeding from point $a$ to point $b$, allows the acquisition of images of the object of study. Subsequently, the images are processed by appropriate automated algorithms aimed at the identification of cracks, and their subsequent visualization and updating through base cartography on the GIS system (Barrile, Bernardo, Fotia, Candela, \& Bilotta, 2020). The acquired data are therefore high resolution images with high spatial sampling rates.

In this research activity, we focused specifically on the analysis of the attributes in the database that can be identified through the classification and segmentation of the images acquired by the proposed system in order to detect the presence of deterioration in the masonry.

\subsection{Innovative measurement system}

We used a fleet of automated drones connected to the cloud (or to a local network) that automatically recharge via special charging stations located at pre-established points (Fig. 2).

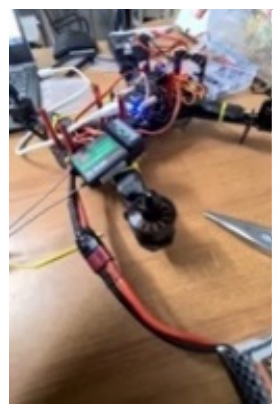

(a)

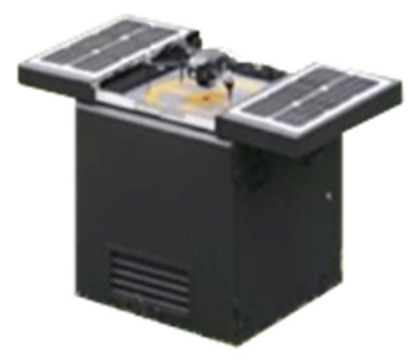

(b)
Figure 2: Automated drones: a) System components; b) Input/output wireless charging base.

The drone fleet sends real time data to the cloud platform, which is then processed by the algorithms to select the images.

The data acquisition system provides for the installation of different platforms along the path to be monitored to allow the drone battery to be recharged and the data necessary for subsequent processing to be transferred.

We have built an innovative monitoring system including drones, intelligent multi-landing and charging pads, automatically governed that communicate at short range with nearby drones and indicate the status of the station.

1) When a drone in flight detects that the battery is running low, it looks for the nearest charging station, the latter communicates to the drone if there is a free pitch and a charged battery available.

2) Having obtained the ok to land, the drone, knowing the GPS coordinates of the station, approaches and, moving vertically, lands on the assigned stand.

3) Once landed, a subsystem recharges the onboard battery or swaps it, automatically replacing the discharged battery with a charged one. During the replacement, the drone is still powered through a special connector in order not to lose communications and to allow the automatic procedure with the exchange of information. After recharging, take-off takes place.

The wireless charging station is made up of an "intelligent" induction plate which, when the drone lands, determines the type of batteries supplied to the aircraft, and thus establishes the correct charging parameters.

This is made possible thanks to a small device on board the drone consisting of a microcircuit with a data transmission system, weighing a few tens of grams and dimensions contained in the order of a few centimeters, such that it can be installed not only on large professional drones but also on smaller commercial ones.

These stations are totally waterproof and weatherproof and also serve as a temporary shelter for appliances. In particular, the process of this first experimentation is divided into three automated phases, schematized in the flow diagram in Figure 3, which include:

1) Definition of the flight plan, in terms of Ground Sampling Distance (GDS), overlapping of images and waypoints.

2) Image analysis: preprocessing - segmentation classification.

3) In order to improve the precision of the image processing and the identification of the investigated element, the use of different cascading algorithms was experimented which involve the combination of different methodologies (segregation; edge detector; Canny filter; Gaussian filter; Support Vector Machine - SVM).

4) Geo-localization of data on the GIS platform.

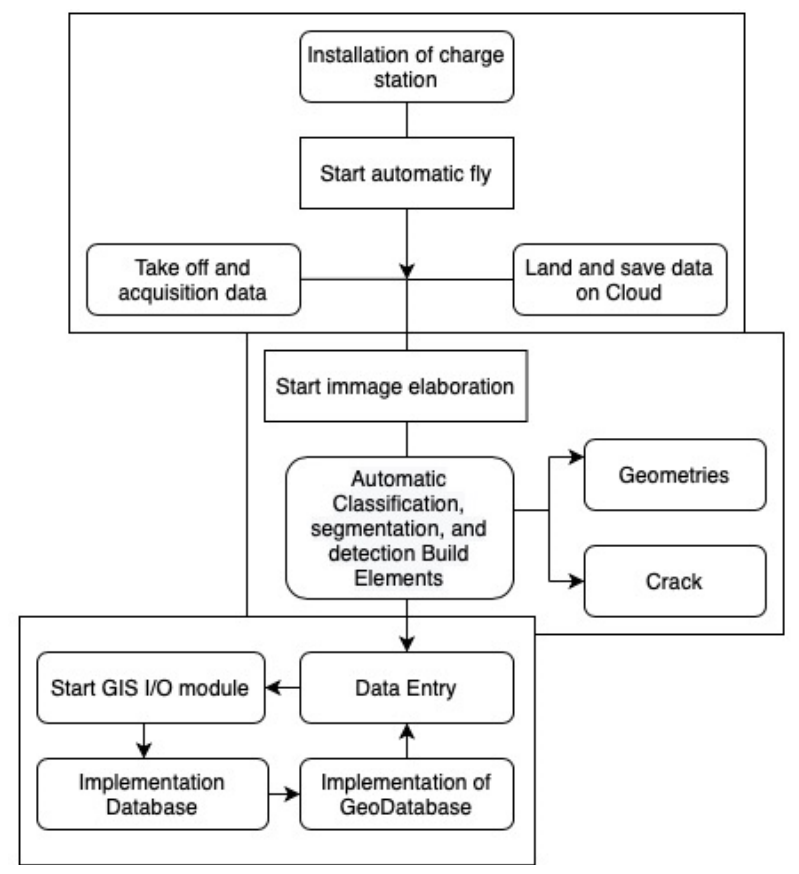

Figure 3: Workflow automatic system GIS integration.

Figure 4 shows the 3D restitution of the village of Casalvecchio Siculo from the study activities carried out by the Geomatics Laboratory as part of the Cultural Heritage (Bernardo \& Bilotta, 2021). By way of example, an image acquired by the system relating to the identification of cracks is shown (Fig. 5). 


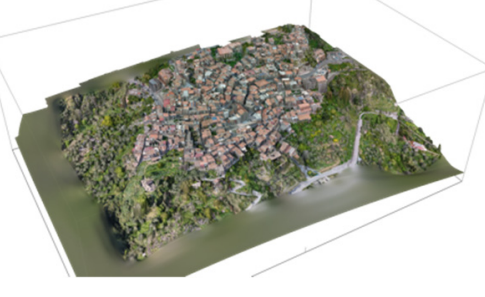

Figure 4: 3D model created.

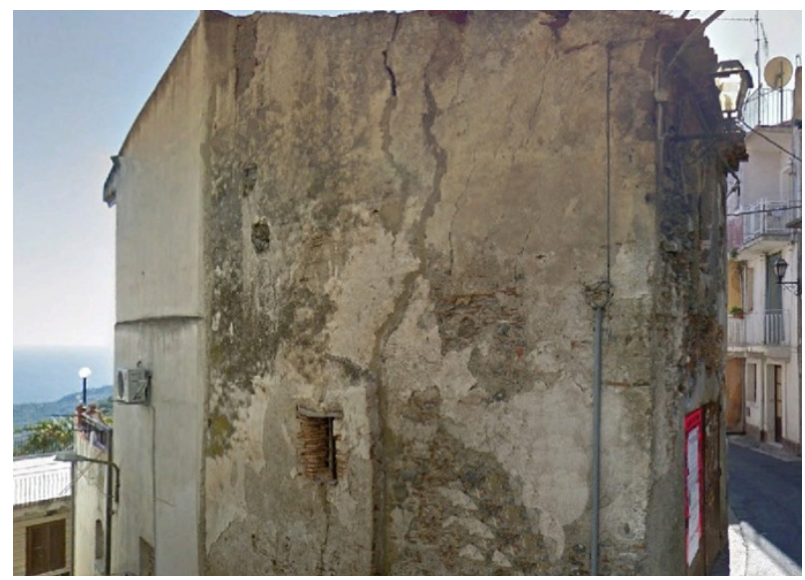

Figure 5: Image acquired by the drone relating to the identification of cracks.

\section{Result and discussion}

\subsection{Image acquisition and processing}

In order to populate the database we have suitably processed the images acquired through the described acquisition system, extracting the geometric characteristics of the buildings, the presence of decay in the walls. To better explain the procedure, an example of methodology applied to the classification of a single type of building crack is reported (Fig. 6).

The acquired images (Fig. 6a) were automatically subjected to a pre-processing and enhancement process (Sobel operator and Prewit), then they were segmented (edge detector, Canny filter, Gaussian filter) and classified (Support Vector Machine) to be able to extrapolate the information we need (Fig. 6b) (Grüen \& Li, 1997; Serna \& Marcotegui, 2014; Ameri, Dadrass Javan, \& Zarrinpanjeh, 2019; Dadrasjavan, Zarrinpanjeh, \& Ameri, 2019). In particular, the SVM classification was carried out in two phases:

1) SVM Training.

2) Performance testing.

In the first phase, the geometric characteristics of the linked components assigned for SVM training were initially calculated. Then, these features were normalized to a range. Kernel with radial base function (RBF) was chosen as a kernel trick, because the number of instances (connected regions) was not very large, and the size of the space transformed with RBF is infinite.
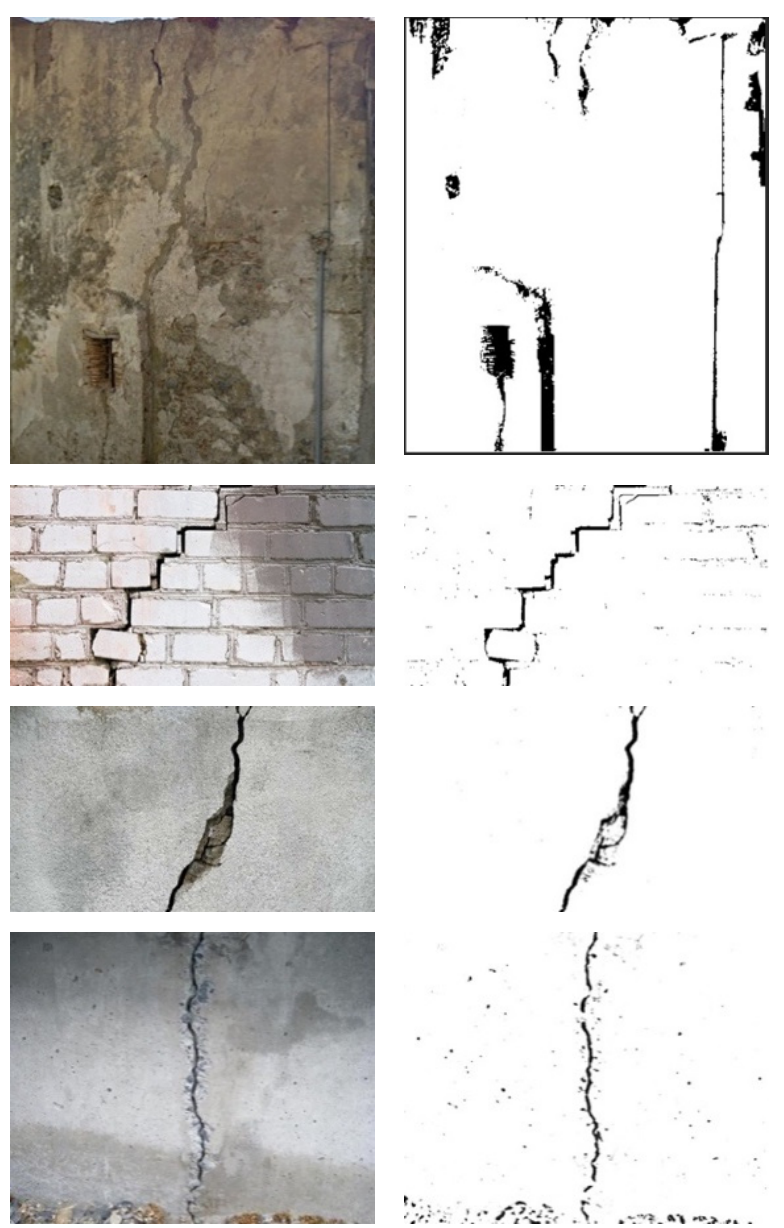

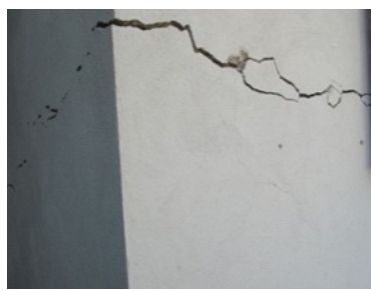

(a)

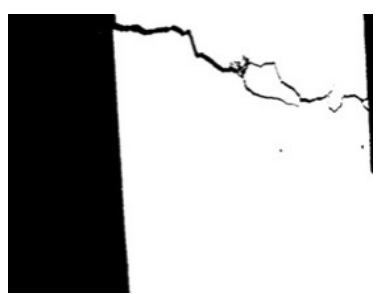

(b)
Figure 6: methodology applied to the classification of a single type of building crack is reported: a) Input Images; b) Crack detection.

The optimal training parameters for the SVM were found using grid search. During this operation, triple crossvalidation was performed to correctly learn the different types of cracks. In this triple cross-validation, the training set was divided into three equal subsets. To ensure proper learning, a subset was tested using the trained classifier on the remaining two subsets. The goal was to identify good parameters so that the classifier can predict test data effectively. After learning that the parameters had been determined, SVM was trained with the "One Against All" approach using the MATLAB LIBSVM library.

In the second phase, connected regions were tested that were not used during SVM training (Grüen \& Li, 1997; Hoang \& Nguyen 2019; Mancini, Malinverni, Frontoni, \& Zingaretti, 2013; Ogawa, Matsushima, \& Takahashi, 2019; Sari, Prakoso, \& Baskara, 2020; Sari, Prakoso, \& Baskara, 2019; Sekeroglu \& Tuncal, K. 2020; Tonkin \& Midgley, 2016). 


\subsection{Big data treatment}

The real value associated with the great availability of data lies not only in the quantity, but in the ability to use them to process, analyze and find objective evidence and correlations between various sectors, in the specific example in favour of the monitoring, inspection and mapping of building cracks at the in order to obtain and constantly update the safety status of buildings through a GIS platform.

Big Data, therefore, means the range of opportunities for analyzing the correlations on the data associated with this aspect, to be used for research, management and conservation applications of cultural heritage. To manage large amounts of data, basic technologies and specific technologies are required.

In this article, we want to describe a solution capable of correlating the large amount of data acquired thanks to the implementation of big data management algorithms. Specifically, these algorithms subsequently reprocess the previously processed images and the acquired data in order to identify if over time there has been an evolution of the crack, otherwise, they are discarded. Algorithms we have used for the treatment of big data (Gopalakrishnan, Gholami, Vidyadharan, Choudhary, \& Agrawal, 2018; Agrawal \& Choudhary, 2016; Bai, 2017; Deng et al., 2009):

- Linear regression (identifies the correlation between the input data and the output data).

- Logistic regression (it is used to give definitive answers: eg. Is the building worthy of attention yes or no?).

- Regression Trees (it is a decision-making system linked to several variables; it allows making a decision for the classification of data relating to different input variables).

- K-nearest neighbours and Kmeans clustering, the first has been used only partially because it is very expensive at the computational level and is affected by both the nature and the type of data to be processed. The second is a fairly fast algorithm at the computation level and presents as well as an easy implementation also an easy training phase, however, it turns out to be very sensitive to anomalous values.

\subsection{Database and Geodatabase}

As is well known, the data in a Geographic Information System are traditionally divided into "graphs" and "attribute". Graphs are organized in vector or raster structures while the attribute data are made up of tables connected to the graphical data and connectable to each other through the rules of the relational databases (through the application of SQL commands on the table structures appropriately). Vector graphic structures are those that mostly use SQL commands for the connection between attribute tables ("select" and "join" commands). Generally, in this way, the thematic cartography is produced, often as an integral part of extensive and indepth territorial studies. In these studies, the term "database" is sometimes used improperly, in the sense that the set of tables connected to graphic layers is defined as a database, very often not connected to each other in any way. A relational database, on the other hand, is a completely different thing or an archive of integrated and shared digital data, through the relationships created by the various keys in the fields of the tables. Storing a multitude of tables connected to graphic layers on a hard disk does not mean building a relational database.

We can define a database as a digital data store. Generally, a software called DBMS (Database Management System) manages databases. The management of a database consists of a whole series of operations for its operation, including data storage, deletion, display (access to the database), updating, processing. As for the data, these are stored only through tables (generally non-square matrices). Tables are made up of columns (fields) and rows (records). The data of a database must be integrated and shared. This feature is of fundamental importance for the correct and efficient functioning of the database.

As mentioned above, integration means avoiding the duplication of a data item in the archive. Integration ensures that information is managed efficiently (for example, updates are faster) and is a good premise to avoid errors within the archive. Data integration is achieved by relating the different tables that populate the database. Relationships are achieved through the equality of common fields ("key" fields).

If geometric components are also stored in the database tables, we can speak of spatial databases or geodatabases. In this way, the database structure acquires additional potential, linked to the spatial analyses (often also very advanced) made available. The management software becomes a "Spatial DBMS", able to perform spatial queries and geographical elaborations of the data obtained from them (Gustavsson, Seijmonsbergen, \& Kolstrup, 2008). Specifically, the database was built with:

- The trajectory graphic subsystem (in ASCII format containing trajectory information), Table 1.

- $\quad$ Photo capture units of the drone (JPEG image folders related to photographic capture, ASCII files containing the connection information between the trajectory data and the images,) Table 2

- What was indicated in the previous paragraph (JPEG image folders related to elaboration, ASCII files containing the connection information between the trajectory data and the elaborated images).

Since there are a multitude of these elements, it is necessary to put them in connection with each other by populating for each of them a table also containing a reference to an element of the upper level. Therefore, the data structure used consists of a set of the integrated relational tables some auxiliary tables that contain the source data and the information processed in progress (Monteiro, Costa, Pina, Santos, \& Ferrão, 2018).

In order to relate the data described above, initial processing is carried out which consists of the population of the table, with the grids and the placement of the 
images within folders with the same name assigned to the measurement session. Copies of the Trajectories table, exported in XML format, and the folders containing the images are then delivered to operators to make observations on frames. For the construction of the Geodatabase, we proceeded by creating and naming the database on pgAdmin and inside it the PostGIS spatial extension was inserted. A connection to the database was built on QGIS in pgAdmin and through the "DB manager" plugin, PostGIS was chosen among the available spatial extensions (thus ensuring the connection between the database and the layers); thus, creating the database tables with spatial component. The experimental system in operation, using the aforementioned automations, was tested on the reference area by acquiring a number of frames equal to 200 , transferred to the cloud and processed. In the post-processing phase, the entities detected automatically were georeferenced and shown on the screen (Fig. 7).

Table 1: Trajectories table.

Trajectories (contains survey data and association with photograms)

Id (primary key)

Id Session (unique identifier assigned to the survey session, and the name of folders containing the image files)

Time (numeric, instant of the measure)

Distance (numeric, odometric distance detected)

Latitude (numeric, latitude)

Longitude (numeric, longitude)

Altitude (numeric)

Heading (numeric)

Frame (string, measure associated file name)

Table 2: Table frame.

Frame (contains the pixel observations performed on the frames, the flat coordinates of the points in the map system and the classification of observations)

$$
\text { Id (primary key) }
$$

Id Session (foreign key to the Trajectories table)

Photogram (foreign key to the Trajectories table)

Typology (main classification numeric code)

Collocation (bin code to the axis [+, -, NULL])

Segmentation (Segment end marking [Start, End, NULL])

Element (numeric, elements road code)

Figure 7 shows the GIS capable of displaying the map of the buildings, the trajectories of the drones, the buildings where the cracks are present (highlighted in yellow). In particular, by clicking on the trajectory we can see all the images acquired, by clicking on the building we are able to identify the crack and the valuable elements.

We realized a first updated map showing the building network updated by the data collected by the proposed experimental system. The buildings are clearly visible from the images and both the presence of signs or artefacts and the conditions of the building surface can be determined. The images acquired by drone clearly show the details of the cracks, and the data can be acquired regularly, without the aid of operators, thus facilitating operations and reducing costs and times.

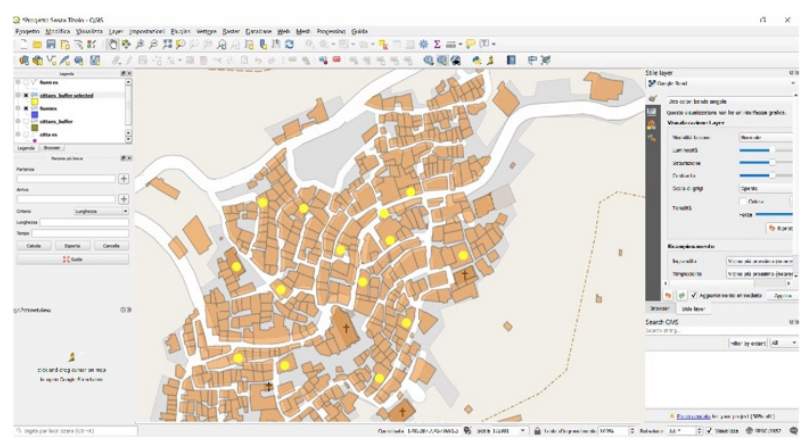

Figure 7: GIS elaboration.

The buildings can then be monitored and consequently facilitate the scheduled maintenance process. A building network geodatabase has been generated. The database shows the coordinate of the buildings identified in the study area, their types and conditions, etc. The study attests to the fact that UAV images can capture more information on the buildings than field investigations and can help in a variety of situations (Barbaro, Fiamma, Barrile, Foti, \& lelo, 2017).

In addition, work is underway on how to make the system usable in accordance with the new EASA regulations.

Moreover, other implementations and therefore additional information can be inserted into the Cadastre of unsafe Buildings. As for the Information System that we are implementing to propose to the municipality of Casalvecchio Siculo, we have populated the dataset with other additional information: census of the segmented attributes of buildings, crack monitoring data. In order to highlight the regions within the study area, that are more distressed from this point of view and then go to assess which are the most appropriate interventions to solve the problem (inserting noise barriers or traffic diversion interventions for example).

In addition, a basic function has been implemented for the planning of maintenance of various aspects such as the state of the masonry, the systems built and the complementary works.

For this purpose, in addition to the geometric and identifying information of the censused elements, the conservative and functional state must also be indicated, thus integrating the information entered in the Cadastre.

\section{Conclusions}

From an economic point of view, the system we created in order to obtain a Cadastre of unsafe buildings is very expensive; however, if implemented with safety, prevention and maintenance functions, it can be an excellent tool for public administrations, which can then plan interventions based on the indications contained within the GIS.

In fact, the Cadastre of buildings can be used with the sole purpose of taking a census of the existing building heritage of the villages. However, based on the acquired data, we could extend the knowledge to elements more aimed at planning maintenance activities such as the 
identification of buildings with a high level of danger (due to the presence of dangerous cracks) and the planning of interventions to increase safety. The databases were then enriched with a series of possible additional information useful for this purpose. It was therefore decided to analyze how to implement the cadastre of buildings in order to be able to use it for planning maintenance and restoration interventions.

Future developments will be aimed at the implementation of automation systems.

\section{Acknowledgements}

We thank the Geomatics laboratory of the Mediterranean University of Reggio Calabria in the person of Prof. Eng. Vincenzo Barrile (scientific and technical manager of the laboratory) and Eng. Antonino Fotia, for support in the complex and different phases of the entire process covered by the $\mathrm{PhD}$ thesis of one of the authors of the article.

\section{References}

Agrawal, A., \& Choudhary, A. (2016). Perspective: Materials informatics and big data: Realization of the "fourth paradigm" of science in materials science. APL Materials 4(5), 1-9. https://doi.org/10.1063/1.4946894

Ameri, A., Dadrass Javan, F., \& Zarrinpanjeh, N. (2019). Automatic Pavement Crack Detection Based on Aerial Imagery. Journal of Geomatics Science and Technology, 9(1), 145-160

Bai, S. (2017). Growing random forest on deep convolutional neural networks for scene categorization. Expert Systems with Applications, 71. 279-287

Barbaro, G., Fiamma, V., Barrile, V., Foti, G., \& lelo, G. (2017). Analysis of the shoreline changes of Reggio Calabria (Italy). International Journal of Civil Engineering and Technology, 8(10), 1777-1791.

Barrile, B., Bernardo, E., \& Fotia, A. (2020). GPS/GIS system for updating capable faults in the Calabrian territory through the use of soft computing techniques. European Association of Geoscientists \& Engineers. Conference Proceedings, International Conference of Young Professionals «GeoTerrace-2020», 2020 (1), p.1-5. https://doi.org/10.3997/22144609.20205710

Barrile, V., Bernardo, E., Fotia, A., Candela, G., \& Bilotta, G. (2020). Road safety: Road degradation survey through images by UAV. WSEAS Transactions on Environment and Development, 16, 649-659, ISSN: $2224-3496$. https://doi.org/10.37394/232015.2020.16.67

Barrile, V., \& Cotroneo, F. (2006) A software for the automatic update of the road cadastre in the GIS environment. Bulletin of the Italian society of photogrammetry and topography.

Bernardo, E., Barrile, V., \& Fotia, A., (2020). Innovative UAV methods for intelligent landslide monitoring. European Association of Geoscientists \& Engineers Conference Proceedings, International Conference of Young Professionals «GeoTerrace-2020», 2020 (1), 1 - 5. https://doi.org/10.3997/2214-4609.20205713. issn: 2214-4609

Bernardo, E., Barrile, V., Fotia, A., \& Bilotta, G. (2020). Landslide susceptibility mapping with fuzzy methodology. European Association of Geoscientists \& Engineers, Conference Proceedings, International Conference of Young Professionals «GeoTerrace-2020», 2020 (1), 1-5. https://doi.org/10.3997/2214-4609.20205712. issn: 2214-4609

Bernardo, E., \& Bilotta, G. (2021). Monumental Arc 3D Model Reconstruction Through BIM Technology. In: Bevilacqua C., Calabrò F., Della Spina L. (eds) New Metropolitan Perspectives. NMP 2020. Smart Innovation, Systems and Technologies, 178. Springer, Cham. https://doi.org/10.1007/978-3-030-48279-4_148

Bernardo, E., Musolino, M., \& Maesano, M. (2021). San Pietro di Deca: From Knowledge to Restoration. Studies and Geomatics Investigations for Conservation, Redevelopment and Promotion. In: Bevilacqua C., Calabrò F., Della Spina L. (eds) New Metropolitan Perspectives. NMP 2020. Smart Innovation, Systems and Technologies, 178. Springer, Cham. https://doi.org/10.1007/978-3-030-48279-4_147

Chen, C., Seo, H. S., Zhao, Y., Chen, B., Kim, J. W., Choi, Y., \& Bang, M. (2019). Automatic Pavement Crack Detection Based on Image Recognition. International Conference on Smart Infrastructure and Construction, (ICSIC), 361-369. https://doi.org/10.1680/icsic.64669.361

Chen, S., Truong-Hong, L., Laefer, D. F., \& Mangina, E., (2018). Automated Bridge Deck Evaluation through UAV Derived Point Cloud. CERI-ITRN2018, Dublin, Ireland, 735-740.

Dadrasjavan, F., Zarrinpanjeh, N., \& Ameri, A. (2019). Automatic Crack Detection of Road Pavement Based on Aerial UAV Imagery. Preprints, 2019070009. https://doi.org/10.20944/preprints201907.0009.v1

Deng, J., Dong, W., Socher, R., Li, L., Li, K., Fei-fei, L., (2009). Imagenet: A large-scale hierarchical image database. In Proceedings of the 2009 IEEE Conference on Computer Vision and Pattern Recognition (CVPR), $248-255$. https://doi.org/10.1109 / CVPR.2009.5206848

Florkova, Z., Duris, L, Veselovsky, M., Sedivý, S., \& Kovalova, D. (2018). Three-dimensional mobile mapping system and its use in road engineering. MATEC Web Conf., 196(04082). https://doi.org/10.1051/matecconf/201819604082

Gopalakrishnan, K., Gholami, H., Vidyadharan, A., Choudhary, A., \& Agrawal, A. (2018). Crack damage detection in unmanned aerial vehicle images of civil infrastructure using pre-trained deep learning model. International Journal 
for Traffic and Transport Engineering, 8(1), 1-14. http://dx.doi.org/10.7708/ijtte.2018.8(1).01

Gustavsson, M., Seijmonsbergen, A., \& Kolstrup, E, (2008). Structure and contents of a new geomorphological GIS database linked to a geomorphological map - With an example from Liden, central Sweden. Geomorphology, 95(34), 335-349. https://doi.org/10.1016/j.geomorph.2007.06.014

Grüen, A., \& Li, H. (1997). Linear feature extraction with 3-D LSB-snakes. Automatic Extraction of Man-Made Objects from Aerial and Space Images, 2, 287-298.

Hoang, N., \& Nguyen, Q. (2019). A novel method for asphalt pavement crack classification based on image processing and machine learning. Engineering with Computers, 35, 487-498. https://doi.org/10.1007/s00366-018-0611-9

Mancini, A., Malinverni, E. S., Frontoni, E., \& Zingaretti, P. (2013). Road pavement crack automatic detection by MMS images. 21st Mediterranean Conference on Control and Automation, Chania, 1589-1596. https://doi.org/10.1109/MED.2013.6608934

Monteiro, C., Costa, C., Pina, A., Santos, M., \& Ferrão, P. (2018). An urban building database (UBD) supporting a smart city information system. Energy and Buildings, 158, 244-260. https://doi.org/10.1016/j.enbuild.2017.10.009

Ogawa, S., Matsushima, K., \& Takahashi, O. (2019). Efficient Pavement Crack Area Classification Using Gaussian Mixture Model Based Features. International Conference on Mechatronics, Robotics and Systems Engineering (MoRSE), Bali, Indonesia, 75-80. https://doi.org/10.1109/MoRSE48060.2019.8998713

Postorino, M.N., Barrile, V., \& Cotroneo, F. (2006). Surface movement ground control by means of a GPS-GIS system. Journal of Air Transport Management, 12(6), 375-381. https://doi.org/10.1016/j.jairtraman.2006.09.003

Sari, Y., Prakoso, P. B., \& Baskara, A. R. (2020). Application of neural network method for road crack detection. TELKOMNIKA, Telecommunication, Computing, Electronics and Control, 18(4), 1962-1967. https://doi.org/10.12928/TELKOMNIKA.v18i4.14825

Sari, Y., Prakoso P. B., \& Baskara, A. R. (2019). Road Crack Detection using Support Vector Machine (SVM) and OTSU Algorithm. $6^{\text {th }}$ International Conference on Electric Vehicular Technology (ICEVT), Bali, Indonesia, $349-354$. https://doi.org/10.1109/ICEVT48285.2019.8993969

Sekeroglu, B., \& Tuncal, K. (2020). Image Processing in Unmanned Aerial Vehicles. Al-Turjman F. (eds) Unmanned Aerial Vehicles in Smart Cities. Unmanned System Technologies, Springer, Cham, 167-179. https://doi.org/10.1007/9783-030-38712-9_10

Serna, A., \& Marcotegui, B. (2014). Detection, segmentation and classification of 3D urban objects using mathematical morphology and supervised learning. ISPRS J. Photogrammetry Remote Sens. 93, 243-255.

Shan, J., \& Toth, C. K. (2018). Topographic Laser Ranging and Scanning: Principles and Processing. Second Edition. CRC Press, Taylor \& Francis Group.

Tonkin, T. N., \& Midgley, N. G. (2016). Ground-Control Networks for Image Based Surface Reconstruction: An Investigation of Optimum Survey Designs Using UAV Derived Imagery and Structure-from-Motion Photogrammetry. Remote Sens., 8(9), 786. https://doi.org/10.3390/rs8090786 